\title{
Pain in the Neck: Are We Potentially at Risk?
}

\author{
Shawn S. Adibia, b, Ezinne I. Ogburekea, Kalu U. Ogburekea, \\ Thomas A. Servos ${ }^{\mathrm{a}}$
}

\begin{abstract}
Clinical symptoms of the patient with Eagle syndrome (ES) are usually very subtle and often confusing even for the most seasoned clinicians. This confusion may lead to a vast number of misdiagnoses. When an elongated styloid process becomes symptomatic, usually the symptoms are varied for each individual, due to the complex nature of the head and neck region. It is essential that a diagnosis of ES be included when evaluating many head and neck conditions. Some cases might require further extensive examination, since ES presents with various heterogeneous symptoms, making it extremely challenging to diagnose. Recently, a case was reported of a sudden death due to ES, a mechanical irritation of the carotid sinus initiated by an elongated styloid process that led to an acute cardiovascular failure. These types of incidences are rare, but very concerning. An elongated styloid process, found frequently in panoramic radiographs, may be a significant and coincidental finding that could possibly be related to many systemic conditions. Therefore, due to calcification of the styloid process, medical attention including a workup would be warranted. A digital panoramic radiograph, readily available in many dental offices, is sufficient to confirm a diagnosis of ES. In rare cases, surgical intervention may become necessary. A cone beam computed tomography $(\mathrm{CBCT})$, which reconstructs a three-dimensional image, can accurately measure the length of the elongated styloid process and assess the severity of the condition. Clinicians, who examine and provide care for the patient with pain in the head and neck region, must become aware of this entity when suspected symptoms are present.
\end{abstract}

Keywords: Temporomandibular disorders; Glossopharyngeal neuralgia; Eagle syndrome; Trigeminal neuralgia; Migraine type headaches; Temporal arteritis; Cervical arthritis; Cervicogenic pain; Carotid artery distention; Trauma; Pain; Diagnosis; Treatment plan

\section{Introduction}

Eagle syndrome (ES) is also known by some as with many

Manuscript submitted May 15, 2017, accepted May 23, 2017

${ }^{a}$ The University of Texas School of Dentistry at Houston, Houston, TX, USA ${ }^{b}$ Corresponding Author: Shawn S. Adibi, The University of Texas School of Dentistry at Houston, 7500 Cambridge Street, Suite 5429, Houston, TX 77054, USA.Email: shawn.adibi@uth.tmc.edu

doi: https://doi.org/10.14740/jmc2848w other designations such as styloid syndrome, carotid artery syndrome or stylalgia. Due to its anatomical location, the styloid process from inferior aspect of temporal bone to slightly anterior to stylomastoid foramen, it is in proximity with many vital structures of neck. That includes external and internal carotid arteries. As it attaches to hyoid bone, it will have to be mobile while patient rotates neck to all directions. Styloid process ability to stretch and flex while patient rotates neck to right and left directions becomes essential to dynamics of neck movement. Mineralization of styloid process is not a usual phenomenon for many. It appears with varied degree with different patients. It restricts patients' movements and puts pressures and compression on adjacent vital nerves and vasculatures. Although it is often a bilateral entity, it affects one side more than the other, usually palpable easily in tonsillar fossa. Symptoms often consist of one or many of inexpressible facial pain, pain while swallowing, opening mouth wide, and turning head in any direction, dysphagia, dysphonia, headaches of unknown origin, unexplained dizziness, otalgia and even sometimes syncope. A typical ES occurs with a history of tonsillectomy, involving cranial nerves V, VII, IX and X. When a mineralized styloid process fractures due to trauma, diagnosis is obvious. However, challenging scenarios are when referral pain involves other head and neck areas.

\section{Case Report}

A 49-year-old Caucasian man presented to the assessment clinic with a chief complaint of "I have trouble chewing food and I need to figure out what is wrong with me". Upon completion of his medical and dental history, all vital signs were within the normal limits. His past and present medical history is significant for borderline diabetes, weight loss during the past year without the need to take weight loss medication, and history of a tonsillectomy. Patient expressed concerns that during the past 3 weeks, he had been experiencing sudden jolt of pain when he opened to bite his food and it eased up slowly as he continued to chew. He experienced stiffness and a tight neck with bilateral pain in the areas of the temples along with frequent headaches and occasional toothaches. Clinical examination of his head and neck area was done, including screening for all cranial nerves. Intraoral and extraoral evaluations revealed no significant abnormal finding. He also experienced severe pain to light bilateral palpations of the neck anterior to the sternocleidomastoids muscles in a horizontal level to the angle of the mandible. Pain also exhibited on bilateral palpation of the pre-auricular areas of the 
temporomandibular joints anterior to tragus of the ear (Fig. 1a, b). A panoramic radiograph revealed a loss of maxillary dentition due to severe caries, no posterior occlusal support, normal bone structures of the condylar heads and articular eminences along with the possibilities of fractures to the bilateral elongated and calcified styloid process (Fig. 2). Due to the severity of pain and the seriousness of this condition, a medical consultation with an ear, nose, and throat specialist should be initiated.

\section{Differential diagnosis}

Based on the patient's medical history and his initial clinical findings along with the variability of symptoms presented, several diagnostic possibilities should be considered. These included, but not limited to, temporomandibular disorders (TMDs), glossopharyngeal neuralgia, ES, trigeminal neuralgia, migraine type headaches, temporal arteritis, cervical arthritis and cervicogenic pain; tumors, carotid artery dissection and trauma should be included in any differential diagnosis.

\section{Diagnosis}

A diagnosis of TMDs is not likely due to the fact that primary source of the pain is identified and confirmed on clinical examination to be bilaterally located in the neck region anterior to temporomandibular joints. TMDs are usually comprised of muscular component disorders, arthralgia, intra-articular joint disorders or degenerative disc disorders. Clinical examination of this patient did not indicate intra-articular disorder findings, muscle pain or tenderness and no evidence of clicking or crepitation of the joints was found. Therefore, TMD as a primary diagnosis can be eliminated.

Glossopharyngeal, trigeminal and other types of neuralgias are usually present with a unilateral lancinating and sharp electric shock type pain that precipitates spontaneously without a known stimulus. As with this case, pain was produced when
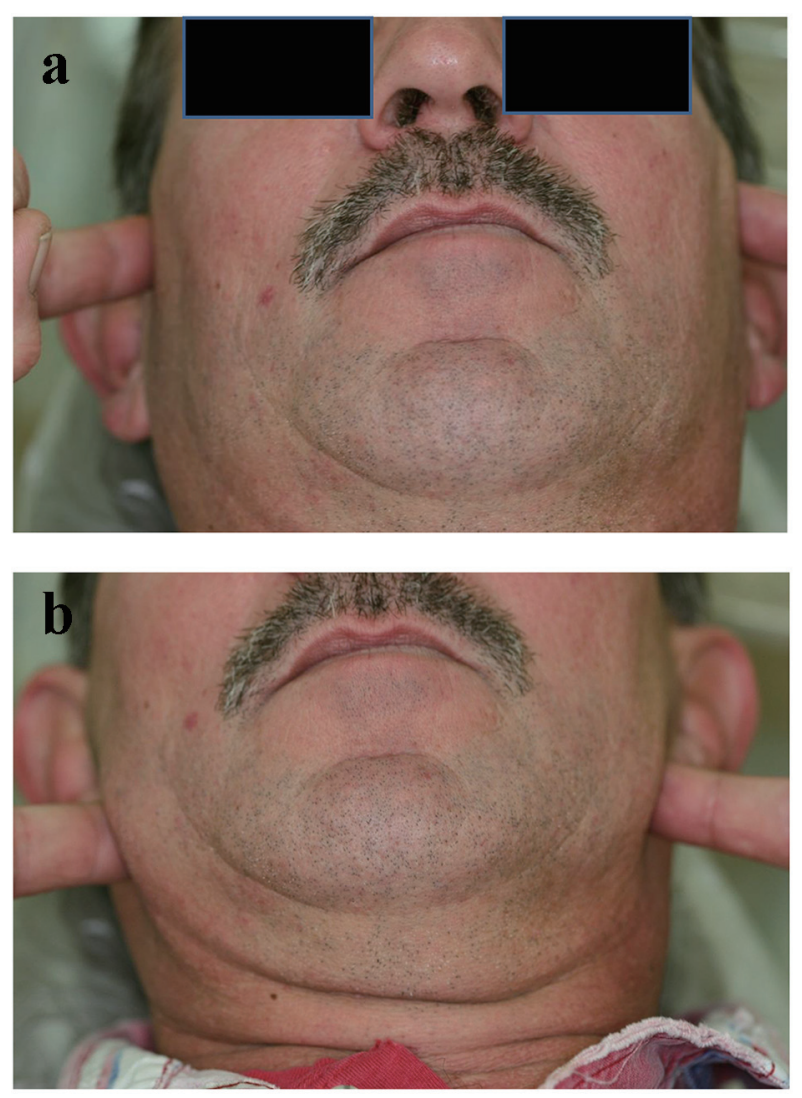

Figure 1. (a) Patient is pointing to areas of slight pain. (b) Patient is pointing to areas of severe pain.

palpating the patient's areas of concern, i.e., the face and neck. Pain also presented when the patient opened for biting and chewing of food, along with some limited neck movements. Additionally, a cranial nerve screening proved negative for any nerve involvement. Therefore, due to the patient's symptoms, typical neurological disorders may be ruled out $[1,2]$.

Migraine type headaches, no matter with or without aura, usually present with a gradual build-up of pain within 5 - 20

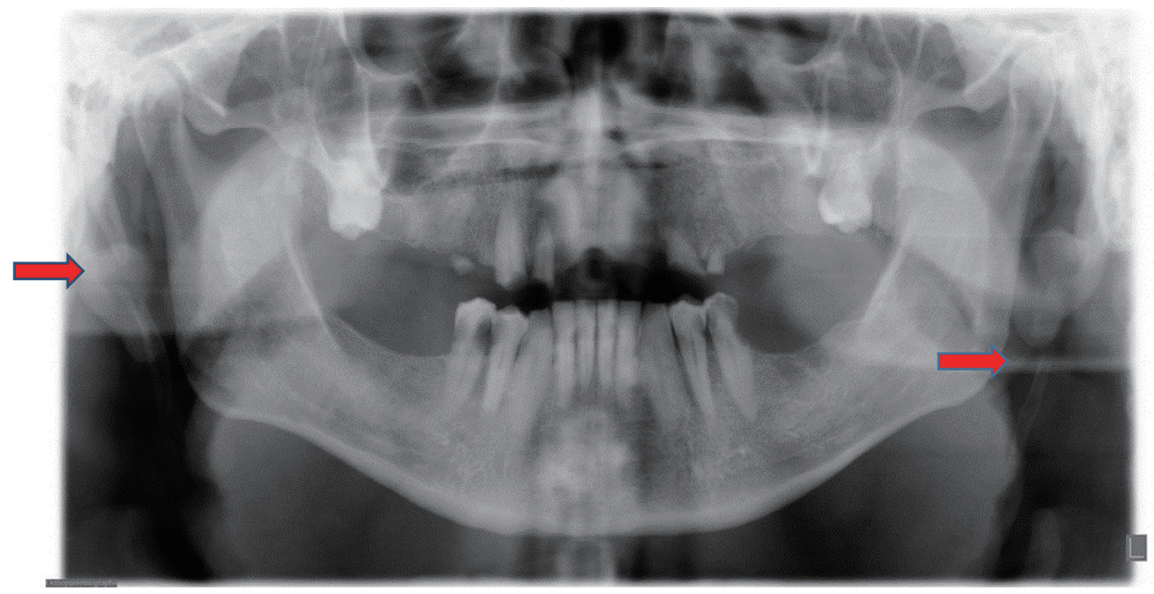

Figure 2. Panoramic image: red arrows indicate fracture points with a break in the calcification process. 


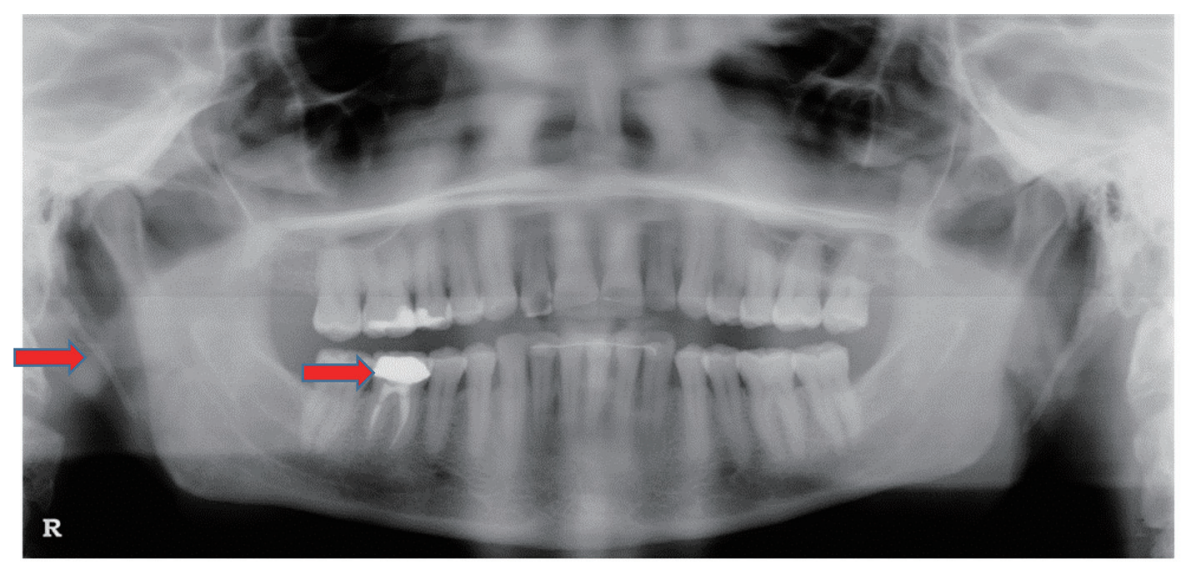

Figure 3. Panoramic image of another patient with a pain complaint concerning tooth \#30. Note: Elongated styloid process with Eagle syndrome.

min and can last in excess of an hour or more. The migraine attack is usually unilateral, pulsating moderate to severe accompanied by nausea. Therefore, due to this patient's findings, migraine headaches may be ruled out. Other possible conditions such as cluster or tension type headaches may be ruled out due to the location of pain experienced by this patient.

Temporal arteritis, also known as giant cell arteritis or cranial arteritis, is considered multifocal vasculitis of temporal arteries. Women above the age of 50, with an average age of 70 , are more affected. This condition is usually accompanied with a burning and throbbing sensation of pain in the temporal area of the head along with a unilateral pulsatile systolic rhythm [3]. Therefore, we can confidently conclude that temporal arteritis is not a diagnosis for this patient.

Cervicogenic conditions are known to produce and refer pain to several areas of the face. A screening exam of the normal movements of the neck is commonly used to reproduce the cervicogenic pain. Cervicogenic pain is usually due to trauma, which could be either acute or chronic, in the absence of any pathology. A comprehensive history and clinical exam of this patient revealed a negative finding of any trauma to the neck and face. Although the patient exhibited mild stiffness of the neck and had endured 3 weeks of pain, there was no familiar pain reproduced within range of motion. Again, cervicogenic disorders are not a likely diagnosis for this patient [1].

Carotid artery distensions are usually a unilateral condition due to the tearing of the carotid artery caused by trauma. They are often diagnosed, in conjunction with a stroke, with the initial signs and conditions such as headaches, drooping of the eyes, vision disturbances and weakness to one side of the body. Due to negative history of trauma and symptoms presented, it is safe to rule out this condition.

A thorough extraoral and intraoral head and neck exam, with palpation, did not reveal any hard or soft tissue abnormalities including tumors. Because of the symptoms presented in the history including tonsillectomy, location of the primary origin of the pain and the ability to reproduce and confirm the familiar pain in that region, ES is a likely diagnosis for this patient due to the elongated and fractured styloid process revealed in this patient's panoramic radiograph.

\section{Management}

Treatment of ES depends on its severity. In many cases, treatment is limited to palliative care, educating the patient and reassurance of their condition [3]. When the condition turns severe, the patient may require a range of pharmacological interventions such as over the counter NSAIDs, transpharyngeal injections of corticosteroids or analgesics to relief pain and inflammation. Rarely, extraoral or intraoral surgical approaches will be needed [4]. It has been reported that there is a $20 \%$ chance of this residual pain being persistent even after surgical interventions [5]. The authors recommend a conservative approach consisting of NSAIDs and patient reassurance in coordination with a medical workup. If not successful, other more aggressive options are available.

\section{Discussion}

The styloid process is a cylindrical, 20 - $30 \mathrm{~mm}$ long cartilaginous bone located on the temporal bone immediately posterior to mastoid apex. It is also adjacent to the carotid artery, the internal jugular vein, the facial nerve, the glossopharyngeal nerve, the vagal nerve and the hypoglossal nerve. It is also attached to muscles and ligaments connected to tongue and throat. Pain modulations, from this area of neck, can refer to the face and is called ES. This condition can only be known as ES when it is symptomatic to facial structures. When styloid process becomes symptomatic, inflamed pain is produced by protrusion of tongue or by turning of the head [6]. An asymptomatic elongated styloid process found frequently in panoramic radiographs may be a significant and coincidental finding that leads the clinician to further investigate the possibilities of related systemic conditions (Figs. 3 and 4). Due to a variation in symptoms and the complexity of the head and neck anatomy, clinicians encounter a diagnostic challenge. 


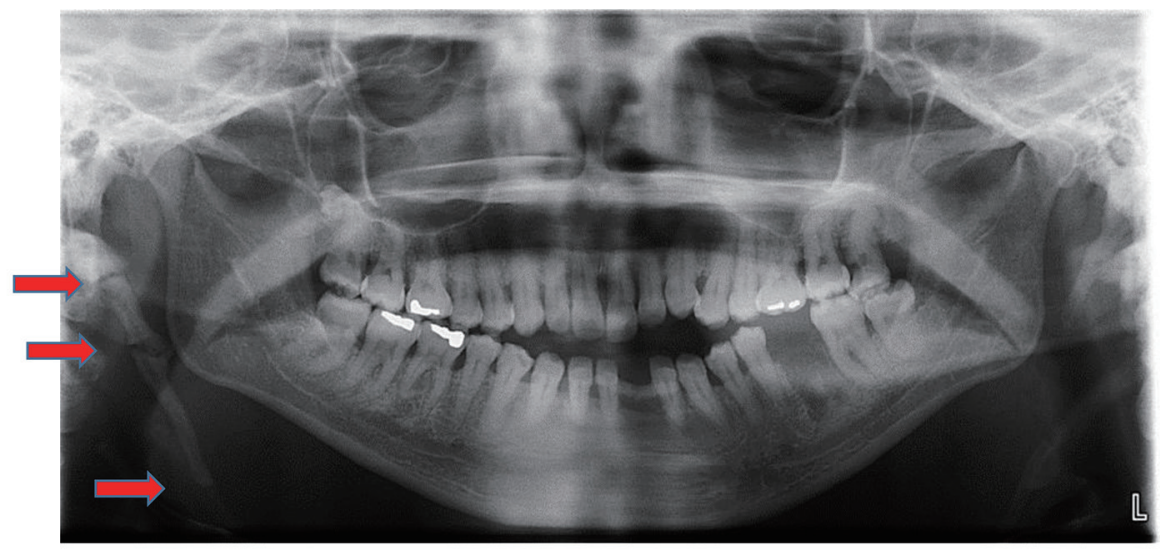

Figure 4. A case of an extremely calcified right styloid process. Red arrows indicate fracture points.

When encountering these clinical challenges, a broad and comprehensive list of differential diagnoses becomes necessary. The etiology of this syndrome is not clear and has been the subject for debate for many years. However, some hypothesized that it is due to compression of inflammatory styloid process on neural and vascular structures of the neck, with a risk of the stroke leading to the compression of the carotid arteries [7]. Additionally, repeated local irritants, surgical trauma, menopausal patients and endocrine disorders are listed as possible etiologies for this syndrome. Recently, Kumar et al reported a case of sudden death due to ES. This death was reportedly caused by the mechanical irritation of the carotid sinus adjacent to an elongated styloid process leading to acute cardiovascular failure [8]. Therefore, medical attention is necessary when this condition is suspected. Gokce et al published the need to study the association between ectopic calcification of styloid process and abnormal serum calcium and potassium levels. These abnormal levels are commonly seen in patients with end-stage renal diseases. Therefore, a clinician must make certain to familiarize him/herself with abnormal radiologic features in order to confirm a diagnosis [9]. A digital panoramic radiograph is readily available in many dental offices and should be sufficient to confirm a diagnosis of ES. However, in some rare cases, when surgical options are planned or other conditions must be ruled out, CBCT should be used. A CBCT reconstructs a three-dimensional image of the anatomy of the affected area and enables the clinician to make an accurate and necessary measurement of the length of the elongated styloid process. Consequently, it will reduce the potential iatrogenic intra-operative injury when surgery is planned [10].

\section{Disclosures}

Authors are supported by the Departments of Diagnostic and Biomedical Sciences, General Practice and Dental Public Health.

\section{Conflicts of Interest}

They report no conflicts of interest.

\section{References}

1. Poon MC, Di Minno G, d'Oiron R, Zotz R. New insights into the treatment of Glanzmann Thrombasthenia. Transfus Med Rev. 2016;30(2):92-99.

2. Okeson JP. Bell's Orofacial Pains: The clinical management of orofacial pain, sixth Edition. Chicago: Quintessence. 2005; p. 364.

3. Neville BW, Damm DD, Allen CM, Bouquot JE. Oral and maxillofacial pathology. Third Edition. St. Luis: Sanders. 2009;24:871.

4. http://radiopaedia.org/articles/eagle-syndrome.

5. Hu M. [Three-dimensional printing and oral medicine]. Zhonghua Kou Qiang Yi Xue Za Zhi. 2017;52(4):206211.

6. Hecquet J, Legaye J, Duval-Beaupere G. Access to a three-dimensional measure of vertebral axial rotation. Eur Spine J. 1998;7(3):206-211.

7. Ceylan A, Koybasioglu A, Celenk F, Yilmaz O, Uslu S. Surgical treatment of elongated styloid process: experience of 61 cases. Skull Base. 2008;18(5):289-295.

8. http://www.rightdiagnosis.com/e/eagles_syndrome/intro. htm.

9. Gokce C, Sisman Y, Sipahioglu M. Styloid process elongation or eagle's syndrome: is there any role for ectopic calcification? Eur J Dent. 2008;2(3):224-228.

10. Kumar P, Rayamane AP, Subbaramaiah M. Sudden death due to Eagle syndrome: a case report. Am J Forensic Med Pathol. 2013;34(3):231-233.

11. https://ispub.com/IJRA/8/1/5142.

12. Savranlar A, Uzun L, Ugur MB, Ozer T. Three-dimensional CT of Eagle's syndrome. Diagn Interv Radiol. 2005;11(4):206-209. 